\title{
Pulmonary vein stenosis: Severity and location predict survival after surgical repair
}

\author{
Mauro Lo Rito, MD, ${ }^{\mathrm{a}, \mathrm{c}}$ Tamadhir Gazzaz, MD, ${ }^{\mathrm{b}}$ Travis J. Wilder, MD, ${ }^{\mathrm{a}, \mathrm{c}}$ Rachel D. Vanderlaan, MD, PhD, ${ }^{\mathrm{a}, \mathrm{c}}$ \\ Glen S. Van Arsdell, MD, ${ }^{\mathrm{a}, \mathrm{c}}$ Osami Honjo, MD, PhD, ${ }^{\mathrm{a}, \mathrm{c}}$ Shi-Joon Yoo, MD, PhD, ${ }^{\mathrm{a}, \mathrm{b}}$ and \\ Christopher A. Caldarone, $\mathrm{MD}^{\mathrm{a}, \mathrm{c}}$
}

\begin{abstract}
Objectives: Pulmonary vein characteristics that influence survival after repair of stenosis have not been defined. We sought to develop a predictive model relating postrepair survival to preoperative pulmonary vein characteristics on computed tomography and magnetic resonance imaging.
\end{abstract}

Methods: Patients who underwent pulmonary vein stenosis repair (1990-2012) with preoperative computed tomography and magnetic resonance imaging were reviewed. We measured pulmonary vein short and long cross-sectional diameters at the left atrial junction (downstream), vein bifurcation (upstream), and narrowest point, and calculated the total cross-sectional area indexed for body surface area. The relationship between pulmonary vein dimensions and survival was related via risk-adjusted parametric hazard analyses.

Results: Of 145 patients who underwent surgical repair, 31 had preoperative computed tomography and magnetic resonance imaging and were analyzed. Surgical repairs were sutureless $(\mathrm{n}=30)$ or pericardial patch reconstruction $(\mathrm{n}=1)$. Mean follow-up was $4.28 \pm 4.2$ years. In-hospital mortality was $9.7 \%$; unadjusted survival was $75 \% \pm 7 \%, 69 \% \pm 8 \%$, and $64 \% \pm 7 \%$ at 1,3 , and 5 years, respectively. Median downstream total cross-sectional area indexed for body surface area was $163 \mathrm{~mm}^{2} / \mathrm{m}^{2}$, upstream total cross-sectional area indexed for body surface area was $263 \mathrm{~mm}^{2} / \mathrm{m}^{2}$, and total cross-sectional area indexed for body surface area at maximal stenosis, localized at the left atrial junction in approximately two thirds of patients, was $163 \mathrm{~mm}^{2} / \mathrm{m}^{2}$. Smaller upstream total cross-sectional area indexed for body surface area $(P=.030)$ and greater number of stenotic pulmonary veins $(P=.0069)$ were associated with increased early $(<1$ year) risk of death. Smaller downstream total cross-sectional area indexed for body surface area tended to be associated with a late risk of death $(P=.059)$.

Conclusions: Smaller upstream or downstream total cross-sectional area indexed for body surface area negatively influenced survival. Early survival seemed especially poor for patients with a greater number of stenotic veins and upstream pulmonary vein involvement. The total cross-sectional area indexed for body surface area measurements can help to inform prognosis and stratify patients for enrollment in clinical trials of agents directed at pulmonary vein pathology. (J Thorac Cardiovasc Surg 2016;151:657-66)

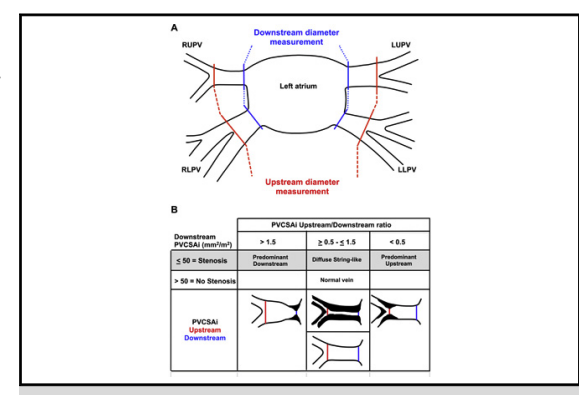

Scheme of PV diameters, measurements, and stenosis classification.

\section{Central Message}

Survival for patients with PV stenosis can be predicted using the upstream/downstream dimensions and the number of involved veins.

\section{Perspective}

The current study introduces an innovative methodology to assess PV stenosis on the basis of CT/MRI. Severity of upstream narrowing and greater number of stenotic PVs are associated with early mortality, whereas the severity of downstream narrowing is associated with late attrition. The proposed methodology could aid in risk stratification for future clinical trials

See Editorial Commentary page 667.

See Editorial page 618.

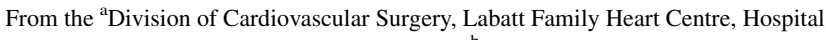
for Sick Children, Toronto, Ontario, Canada; ${ }^{\mathrm{b}}$ Department of Diagnostic Imaging, Hospital for Sick Children, Toronto, Ontario, Canada; and ${ }^{\mathrm{c} D e p a r t m e n t ~ o f ~ S u r g e r y, ~}$ University of Toronto, Toronto, Ontario, Canada.

Read at the 95th Annual Meeting of The American Association for Thoracic Surgery, Seattle, Washington, April 25-29, 2015.

Received for publication April 21, 2015; revisions received Aug 25, 2015; accepted for publication Aug 29, 2015; available ahead of print Oct 16, 2015.
Address for reprints: Christopher A. Caldarone, MD, Division of Cardiovascula Surgery, Labatt Family Heart Center, Hospital for Sick Children and University of Toronto, 555 University Ave, Toronto, ON, Canada M5G1X8 (E-mail: chris. caldarone@sickkids.ca).

$0022-5223 / \$ 36.00$

Copyright $(C) 2016$ by The American Association for Thoracic Surgery http://dx.doi.org/10.1016/j.jtcvs.2015.08.121 


\section{Abbreviations and Acronyms \\ $\mathrm{CT}=$ computed tomography \\ IQR = interquartile range \\ LLPV = left lower pulmonary vein \\ LUPV = left upper pulmonary vein \\ MRI = magnetic resonance imaging \\ PV = pulmonary vein \\ PVCSAi $=$ pulmonary vein cross-sectional area indexed to body surface area \\ RLPV = right lower pulmonary vein \\ RUPV = right upper pulmonary vein \\ TCSA $=$ total pulmonary vein cross-sectional area \\ TCSAi $=$ total pulmonary vein cross-sectional area indexed to body surface area}

\section{Scanning this QR code will take you to the article appendix and supplemental tables. To view the AATS 2015 Webcast, see the URL at the end of the article.}

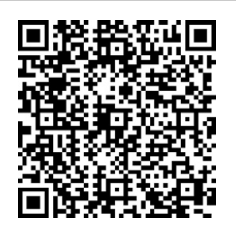

Pulmonary vein (PV) stenosis is common after repair of total anomalous pulmonary venous drainage, affecting $10 \%$ to $17 \%$ of the patients. ${ }^{1-3}$ Less frequently $(0.4 \%-0.6 \%)$, PV stenosis presents as a primary congenital disease. ${ }^{4,5}$ In either form - postrepair or primary congenital disease - the overall outcomes remain poor. Furthermore, surgical intervention does not substantially improve outcomes and is associated with relatively high rates of mortality $(46 \%-80 \%){ }^{1,4-9}$ Available scoring systems created to predict postoperative survival are based mainly on echocardiogram measurements. ${ }^{4,10,11}$ However, these scoring systems are limited by the inability to image the PVs "upstream" within the lung parenchyma. We hypothesized that the upstream PV dimensions, evaluable precisely with computed tomography (CT) and magnetic resonance imaging (MRI), are important determinants of postrepair survival. As such, we used CT and MRI to evaluate the relationship between PV characteristics and survival after surgical repair.

\section{MATERIALS AND METHODS}

We retrospectively reviewed patients who underwent $\mathrm{PV}$ stenosis repair at the Hospital for Sick Children (Toronto, Ontario, Canada) from 1990 to 2012 and who had a preoperative CT or MRI. Among 145 patients treated for PV stenosis, 110 were excluded because they did not have a preoperative $\mathrm{CT} / \mathrm{MRI}$ and 4 were excluded secondary to associated right lung hypoplasia in scimitar syndrome. Of the remaining 31 patients, 16 had a preoperative MRI, 10 had a preoperative CT, and 5 had both MRI and CT imaging before surgery. These 31 patients formed the study population for the subsequent analysis. The Research Ethics Board approved the study and waived the requirement for patient consent.

\section{Data Acquisition and Follow-up}

Patient admission, diagnostic, and surgical data were obtained from medical records, echocardiogram, and surgical reports. Details of PV morphology and physiology were abstracted from CT/MRI reports and operative notes. Follow-up was obtained through review of medical records up to December 2014, with a mean duration of 4.28 years (range, $0.14-12.8$ years).

\section{Imaging Review}

Preoperative PV dimensions were assessed through retrospective analysis of CT/MRI performed by 2 pediatric cardiac radiologists (TG, S-JY) using previously published methods. ${ }^{12-16}$ The data sets were analyzed using offline workstations both for angiographic ("Leonardo," Siemens Medical Solution, Forchleim, Germany or "Advantage Windows 3.0," GE Medical System, Milwaukee, Wis) and flow velocity mapping studies (MEDIS Medical Imaging System, Leiden, The Netherlands). For the subset of patients in whom flow studies were feasible, flow volumes were measured for each PV and for the right/left pulmonary arteries. Pulmonary artery redistribution was considered present when right and left pulmonary artery flow ratio was out of the normality range (between 61:39 and 43:57). ${ }^{14}$

Short and long cross-sectional diameters were measured for each PV at the left atrial junction (downstream) and the bifurcation (upstream) (Figure 1). If the site of maximum narrowing did not include the upstream or downstream location, a third measurement was taken at the narrowest portion of the PV, thus representing the site of maximum stenosis. By applying an ellipse model, the diameters were used to calculate the individual PV crosssectional area indexed to body surface area (PVCSAi) via the Haycock formula. ${ }^{17}$ Total PV cross-sectional area indexed to body surface area (TCSAi) was obtained by adding the single PVCSAi for every vein at the designated level (eg, downstream, upstream, or maximal stenosis).

We also calculated the predicted total pulmonary vein cross-sectional area (TCSA) for each patient using the theoretic derivation formulas for cardiovascular structures in children published by Sluysmans and Colan ${ }^{18}$ based on the power function model $(\mathrm{TCSA}=\mathrm{aX}$ " with an " $\mathrm{a}$ " as the constant value of 1.861 , " $\mathrm{X}$ " equal to body surface area calculated with Haycock formula, and "b" as the exponent with a value of 1.020). The predicted PVCSA was obtained by division of the predicted TCSA by the percentage of contribution of each mean single PV diameter (right upper pulmonary vein $[\mathrm{RUPV}]=25.8 \%$, right lower pulmonary vein $[R L P V]=25.8 \%$, left upper pulmonary vein $[\mathrm{LUPV}]=25 \%$, left lower pulmonary vein $[\mathrm{LLPV}]=23.4 \%$ ) to the total mean diameter.

For illustrative purposes, the PV morphology was classified into 3 groups based on the ratio between the upstream and downstream PVCSAi. An upstream/downstream PVCSAi ratio cutoff of 1.5 and 0.5 were arbitrarily adopted considering that they correspond to vessel diameter variation greater than $20 \%$ to $25 \%$ between the 2 locations. The 3 morphologies possible were as follows: (1) PVs that had localized downstream narrowing with dilated upstream tract and a ratio $>1.5$; (2) PVs that had similar dimensions when comparing the upstream and downstream PVCSAi (ratio 0.5-1.5). This group encompassed normal PVs and veins with string-like stenosis diffusely distributed between the upstream and downstream segments (albeit with clinically evident stenosis). (3) PVs that had a morphology characterized by smaller size upstream and wider size at the left atrial junction (ratio $<0.5$ ).

\section{Statistical Analysis and Presentation}

Demographic, clinical information, and PV dimensions (indexed to body surface area) before surgical repair were included as baseline variables. Continuous variables are summarized as median with interquartile range (IQR). Categoric and ordinal variables are presented as frequencies and percentages. Data analyses were performed with SAS statistical software (version 9.2; SAS Institute, Inc, Cary, NC) and Stata Statistical Software Release 12 (StataCorp LP, College Station, Tex).

The clinical impact of preoperative PV stenosis was determined, in part, by examining the time-related freedom from death (survival) after 


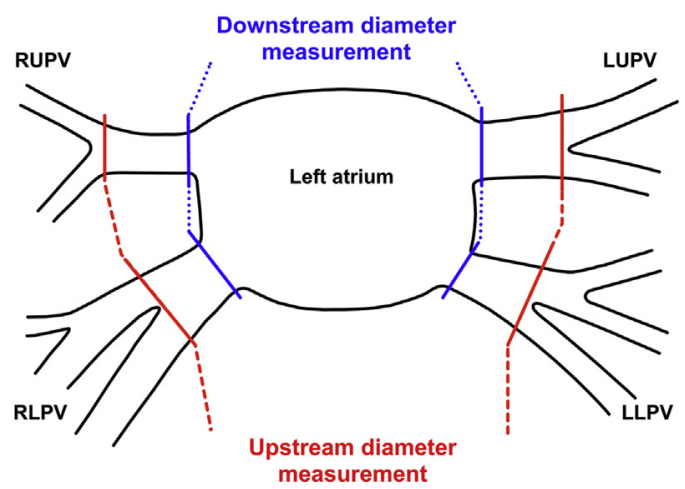

A measurement

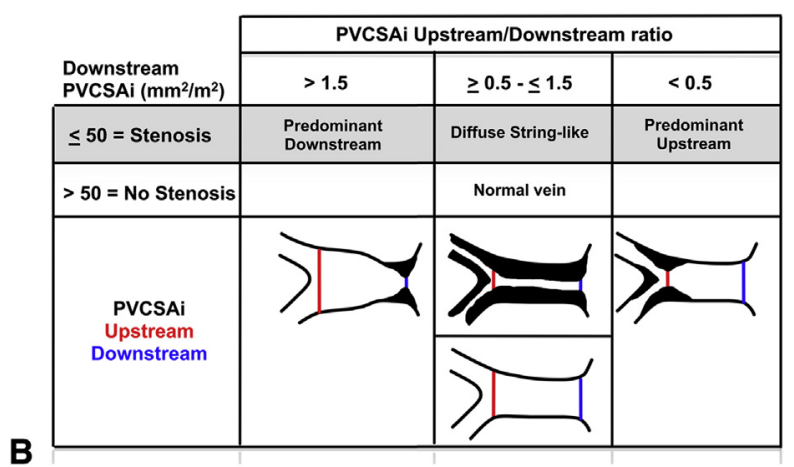

FIGURE 1. Scheme of PV cross-sectional measurements (A) and PV stenosis classification (B). A, The scheme illustrates the upstream (red line) and downstream (blue line) where the diameters are measured by CT/MRI. The long and short diameters are used to calculate single PVCSAi at upstream and downstream levels. The sum of all single PVCSAi provides the TCSAi at the upstream region (red dotted line) and downstream region (blue dotted line). B, In the classification based on PVCSAi upstream/downstream ratio, 3 types of PV morphology can be identified. Predominant downstream narrowing with normal/dilated upstream (ratio $>1.5$ ), diffuse string-like narrowing (ratio, 0.5-1.5), and predominant upstream narrowing with normal/dilated downstream (ratio $<0.5$ ). In case of upstream/downstream ratio between 0.5 and 1.5, the PVCSAi downstream has been used to differentiate between diffuse string-like stenosis and normal vein; a PVCSAi $50 \mathrm{~mm}^{2} / \mathrm{m}^{2}$ or less indicates a stenotic PV, whereas a PVCSAi greater than $50 \mathrm{~mm}^{2} / \mathrm{m}^{2}$ indicates normal PV. RUPV, Right upper pulmonary vein; $L U P V$, left upper pulmonary vein; $R L P V$, right lower pulmonary vein; $L L P V$, left lower pulmonary vein; $P V C S A i$, pulmonary vein cross-sectional area indexed to body surface area.

surgical correction. The time-related freedom from death was assessed nonparametrically by plotting the unadjusted Kaplan-Meier estimates of the time to event. Subsequently, the underlying hazard function was modeled parametrically: assessing the rate of transition from date of surgical repair to death, with patients otherwise censored at the most recent date known to be alive. ${ }^{19}$ These parametric models use shaping parameters that capture various "phases of risk," each of which can be subjected independently to risk hazard regression analysis ${ }^{19}$ (for additional details, see http://my.clevelandclinic.org/professionals/software/hazard/default. aspx). After generation of the "baseline" parametric model, univariate and multivariable risk hazard analyses were conducted incorporating variables for baseline demographic, morphologic, and operative characteristics (Table E1). Final variable selection was guided by bootstrap resampling $^{1}(\mathrm{n}=500$ resamples, threshold for inclusion $P<.1){ }^{20}$ Further details are described in Appendix E1.
TABLE 1. Diagnosis and demographic patient characteristics

\begin{tabular}{|c|c|c|}
\hline \multicolumn{2}{|l|}{ Diagnosis } & No. of patients \\
\hline \multicolumn{2}{|l|}{ PV stenosis isolated } & 7 \\
\hline \multicolumn{2}{|l|}{ PV stenosis associated to heart defect } & 24 \\
\hline \multicolumn{2}{|c|}{ Total anomalous pulmonary venous drainage } & 7 \\
\hline \multicolumn{2}{|l|}{ Hypoplastic left heart syndrome } & 3 \\
\hline \multicolumn{2}{|l|}{ Double-outlet right ventricle } & 4 \\
\hline \multicolumn{2}{|l|}{ Previous heart transplant } & 2 \\
\hline \multicolumn{2}{|c|}{ Atrioventricular septal defect (complete) } & 2 \\
\hline \multicolumn{2}{|c|}{ Tricuspid atresia with transposition of great arteries } & 2 \\
\hline \multicolumn{2}{|l|}{ Partial anomalous PV return } & 1 \\
\hline \multicolumn{2}{|l|}{ Ventricular septal defect } & 1 \\
\hline \multicolumn{2}{|c|}{ Aortic atresia and ventricular septal defect } & 1 \\
\hline \multicolumn{2}{|l|}{ Hypoplastic right ventricle } & 1 \\
\hline Demographic variables & Median & IQR \\
\hline Age (d) & 226 & $130-594$ \\
\hline Weight (kg) & 6.2 & $4.5-10$ \\
\hline BSA at surgery $\left(\mathrm{m}^{2}\right)$ & 0.36 & $0.28-0.46$ \\
\hline BSA at CT/MRI $\left(\mathrm{m}^{2}\right)$ & 0.31 & $0.27-0.45$ \\
\hline Cardiopulmonary bypass time (min) & 107 & $81-134$ \\
\hline Aortic crossclamp time (min) & 63 & $44-91$ \\
\hline $\begin{array}{l}\text { Deep hypothermic circulatory arrest } \\
\text { (min, } 10 \text { patients) }\end{array}$ & 20 & $9-36$ \\
\hline ICU stay (d) & 6 & $2-25$ \\
\hline Hospital stay (d) & 18 & $6-53$ \\
\hline
\end{tabular}

$P V$, Pulmonary vein; $B S A$, body surface area; $C T$, computed tomography; $M R I$, magnetic resonance imaging; $I C U$, intensive care unit.

\section{RESULTS}

\section{Patients' Characteristics}

Among 31 patients analyzed, PV stenosis was associated with other congenital heart defects in 24 (Table 1). There were 20 patients $(65 \%)$ who presented with primary PV stenosis and 11 patients (35\%) who had stenosis secondary to a previous $\mathrm{PV}$ operation. Of these 11 patients, $8 \mathrm{had}$ a total anomalous pulmonary venous drainage repair or PV operation and 1 had hypoplastic left heart syndrome and underwent a hybrid stage I with PV stenosis related to atrial stent-related fibrosis. In addition, 2 patients developed PV stenosis after primary heart transplantation for failing stage 1 palliation and hypoplastic left heart syndrome.

The median age at repair was 226 days (IQR, 130-594 days). Surgical techniques adopted were complete sutureless repair in 18 patients $(58 \%)$, single side sutureless repair in 12 patients $(39 \%)$, and pericardial patch enlargement of the junction between the left atrium and the left PVs in 1 patient (3\%).

\section{Analysis of Preoperative Pulmonary Vein Anatomy}

With the exception of 1 patient ( $3 \%)$, there were multiple PVs that were stenotic or occluded (2 PVs in 52\%, 3 PVs in $10 \%$, and $4 \mathrm{PVs}$ in $35 \%$ ). The stenosis was bilateral in $45 \%$ of patients and unilateral in $55 \%$ of patients, with isolated right-sided vein involvement observed in only 1 patient. The left-sided PVs were far more commonly affected than 
TABLE 2. Cross-sectional areas and flow measurements

\begin{tabular}{|c|c|c|c|c|c|c|c|}
\hline \multirow{2}{*}{$\begin{array}{c}\text { Variables } \\
\begin{array}{c}\text { Cross-sectional area indexed } \\
\text { for BSA }\left(\mathrm{mm}^{2} / \mathrm{m}^{2}\right)\end{array}\end{array}$} & \multicolumn{2}{|c|}{ Total $(\mathbf{n}=\mathbf{3 1})$} & \multicolumn{2}{|c|}{ Alive $(n=20)$} & \multicolumn{2}{|c|}{ Dead $(n=11)$} & \multirow[b]{2}{*}{$P$ value ${ }^{*}$} \\
\hline & Median & IQR & Median & IQR & Median & IQR & \\
\hline RUPV downstream & 51 & $26-90$ & 72 & $37-115$ & 36 & $14-44$ & .046 \\
\hline RLPV downstream & 66 & $31-132$ & 78 & $37-153$ & 41 & $27-81$ & .332 \\
\hline LUPV downstream & 18 & $8-33$ & 21 & $6-60$ & 17 & $16-22$ & .955 \\
\hline LLPV downstream & 31 & $13-43$ & 26 & $8-50$ & 34 & $16-41$ & .628 \\
\hline RUPV upstream & 74 & $55-114$ & 77 & $60-116$ & 45 & $41-104$ & .08 \\
\hline RLPV upstream & 94 & $60-156$ & 100 & $60-155$ & 89 & $39-176$ & .50 \\
\hline LUPV upstream & 50 & $35-75$ & 49 & $18-70$ & 58 & $42-77$ & .425 \\
\hline LLPV upstream & 54 & $33-89$ & 57 & $39-94$ & 46 & $14-61$ & .291 \\
\hline Total PV downstream & 163 & $119-275$ & 233 & $139-284$ & 130 & $96-163$ & .043 \\
\hline Total PV upstream & 263 & $204-415$ & 314 & $219-417$ & 220 & $193-282$ & .186 \\
\hline Total PV narrowest & 163 & $96-232$ & 207 & $139-269$ & 119 & $82-163$ & .023 \\
\hline Aorta & 400 & $181-183$ & 182 & $182-184$ & 181 & $181-182$ & .787 \\
\hline RPA & 159 & $352-479$ & 398 & $363-481$ & 400 & $276-477$ & .589 \\
\hline LPA & 117 & $121-193$ & 164 & $122-194$ & 132 & $111-193$ & .500 \\
\hline MRI blood flow $\left(\mathrm{L} / \mathrm{min} / \mathrm{m}^{2}\right)$ & Tota & $=20)$ & Ali & $=15)$ & Dea & $=5)$ & $P$ value $*$ \\
\hline RUPV & 0.73 & $0.61-0.96$ & 0.74 & $0.61-1.06$ & 0.67 & $0.57-0.73$ & .428 \\
\hline RLPV & 0.95 & $0.73-1.35$ & 0.99 & $0.73-1.35$ & 0.82 & $0.76-1.15$ & .650 \\
\hline LUPV & 0.35 & $0.25-0.84$ & 0.32 & $0.19-0.64$ & 0.73 & $0.46-1.04$ & .089 \\
\hline LLPV & 0.51 & $0.33-0.70$ & 0.51 & $0.30-0.70$ & 0.57 & $0.39-1.13$ & .308 \\
\hline PV total flow & 2.95 & $2.42-3.70$ & 2.95 & $2.42-3.70$ & 3.26 & $2.50-3.73$ & .909 \\
\hline RPA net flow & 1.70 & $1.24-2.15$ & 1.93 & $1.26-2.29$ & 1.34 & $1.02-1.72$ & .365 \\
\hline LPA net flow & 0.63 & $0.31-1.43$ & 0.55 & $0.28-1.02$ & 1.18 & $0.69-1.75$ & .112 \\
\hline RPA perfusion $(\%)$ & 73 & $52-84$ & 81 & $57-86$ & 54 & $49-59$ & .112 \\
\hline LPA perfusion $(\%)$ & 27 & $15-48$ & 19 & $14-43$ & 46 & $41-51$ & .112 \\
\hline
\end{tabular}

Cross-sectional areas and flow measurements for patients who are not known to have died (alive) and for patents who died (dead). BSA, Body surface area; IQR, interquartile range; $R U P V$, right upper pulmonary vein; $R L P V$, right lower pulmonary vein; $L U P V$, left upper pulmonary vein; $L L P V$, left lower pulmonary vein; $P V$, pulmonary vein; $T C S A i$, total cross-sectional area of pulmonary vein indexed to BSA; $R P A$, right pulmonary artery; $L P A$, left pulmonary artery; $M R I$, magnetic resonance imaging. $* P$ values were determined using Mann-Whitney $U$ test and do not account for time-related survival. Bold denotes significant $P$ value.

the right-sided veins, with the LUPV and LLPV involved in $97 \%$ and $93 \%$ of the cases, respectively, and the RUPV and RLPV involved in $48 \%$ and $39 \%$ of the cases, respectively. The site of maximal stenosis was at the left atrial junction in approximately two thirds of the patients. All values of the upstream and downstream PVCSAi are listed in Table 2.

For each single PV, the measured downstream PVCSAi on preoperative CT/MRI was plotted against the predicted downstream PVCSAi calculated with the method of Sluysmans and Colan. ${ }^{18}$ Each PV was then coded stenotic or not on the basis of the definition assigned on CT/MRI and surgical reports (Figure 2). A downstream PVCSAi dimension of $50 \mathrm{~mm}^{2} / \mathrm{m}^{2}$ was identified as a cut-point separating stenotic from nonstenotic PVs. Likewise, a predicted PVCSAi of $47 \mathrm{~mm}^{2} / \mathrm{m}^{2}$ or less seems to be a cut-point separating stenotic from nonstenotic. A downstream PVCSAi $50 \mathrm{~mm}^{2} / \mathrm{m}^{2}$ or less demonstrated good sensitivity $(85 \%)$ and specificity $(82 \%)$ with a positive predictive value of $91 \%$ and a negative predictive value of $70 \%$ for identifying stenotic PVs. The median downstream TCSAi was $163 \mathrm{~mm}^{2} / \mathrm{m}^{2}$ (IQR, $119-275 \mathrm{~mm}^{2} / \mathrm{m}^{2}$ ), the maximal stenotic TCSAi was $163 \mathrm{~mm}^{2} / \mathrm{m}^{2}$ (IQR, 96-
$232 \mathrm{~mm}^{2} / \mathrm{m}^{2}$ ), and the upstream TCSAi was $263 \mathrm{~mm}^{2} / \mathrm{m}^{2}$ (IQR, 204-415 $\mathrm{mm}^{2} / \mathrm{m}^{2}$ ).

In the majority of stenotic PVs (64\%), the upstream/ downstream PVCSAi ratio was greater than 1.5, indicating relative dilatation of the upstream tract. Less commonly, stenotic PVs had a "string-like" morphology (19.8\%) with a ratio between 0.5 and 1.5 . Few PVs $(7 \%)$ had a ratio less than 0.5 , indicating that the narrowing was located mainly in the upstream portion. For $9.2 \%$ of the PVs, it was not possible to define the morphology because they were completely occluded.

\section{Preoperative Flow Analysis}

MRI flow analysis was available for 20 of 31 patients (Table 2). For PVs with a PVCSAi of $50 \mathrm{~mm}^{2} / \mathrm{m}^{2}$ or less, the median blood flow indexed to body surface area was lower than the blood flow in PVs with a larger PVCSAi $\left(0.57\right.$ vs $\left.1.06 \mathrm{~L} / \mathrm{min} / \mathrm{m}^{2}, P<.001\right)$, suggesting that PV stenosis is associated with flow redistribution away from the stenotic veins. Preoperative pulmonary artery flow redistribution was present in 13 patients and absent in the remaining 7 patients. Approximately half of the 


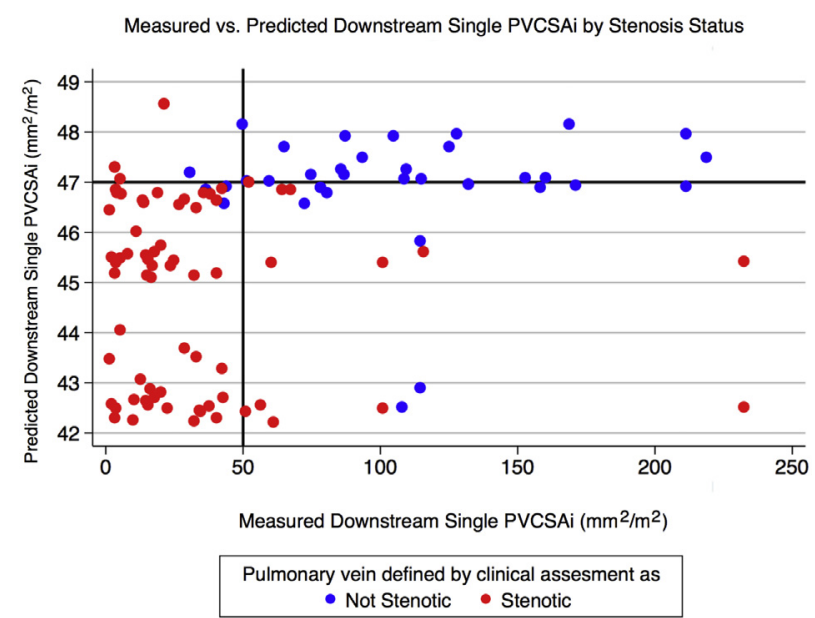

FIGURE 2. Measured downstream single PVCSAi versus predicted downstream single PVCSAi by clinically assessed stenosis status. The measured downstream single PVCSAi is shown in the x-axis. The predicted downstream single PVCSAi obtained with Sluysmans and Colan's predictive model is shown in the y-axis. ${ }^{18}$ Each dot represents a single PV with its measured (x-axis) and predicted (y-axis) PVCSAi value. On the basis of clinical assessment, the blue dots represent nonstenotic PVs and the red dots represent stenotic PVs. The vertical reference black line $\left(50 \mathrm{~mm}^{2} / \mathrm{m}^{2}\right)$ is the stenosis cut-point value determined for the measured downstream PVCSAi, and the horizontal black line $\left(47 \mathrm{~mm}^{2} / \mathrm{m}^{2}\right)$ is the stenosis cut-point value determined for predicted downstream PVCSAi. PVCSAi, Pulmonary vein cross-sectional area indexed to body surface area.

patients $(3 / 7,43 \%)$ without pulmonary artery flow redistribution developed pulmonary hypertension that led to death, whereas only 1 patient with flow redistribution $\operatorname{died}(1 / 13,7.7 \%)$.

\section{Survival}

In-hospital mortality was $9.7 \%$ (3/31), and for 2 patients it was secondary to early recurrence of PVobstruction. In addition, there were 8 late deaths resulting in an overall survival of $75 \% \pm 7 \%, 69 \% \pm 8 \%$, and $64 \% \pm 7 \%$ at 1,3 , and 5 years, respectively (Figure 3). Patients alive at the last follow-up have a significantly larger downstream TCSAi (median: 233 vs $130 \mathrm{~mm}^{2} / \mathrm{m}^{2}, P=.043$ ) and maximal stenotic TCSAi (median: 207 vs $119 \mathrm{~mm}^{2} / \mathrm{m}^{2}, P=.023$ ) compared with nonsurvivors. However, the upstream TCSAi was not different between survivors and nonsurvivors (313 vs $220 \mathrm{~mm}^{2} / \mathrm{m}^{2}, P=.183$ ) (Table 2). Although this comparison between survivors and nonsurvivors cannot be used to determine a causal relationship between pulmonary vessel size and death, it illustrates the important differences between these two groups.

On univariate analysis, a younger age at operation, lower body surface area, smaller upstream TCSAi, and greater number of PV with stenosis/occlusion were associated with an increased risk of death (Table E1).

On multivariable analysis, smaller upstream TCSAi $(P=.030)$ and greater number of stenotic PVs $(P=.007)$

\section{Overall estimated 8 year survival}

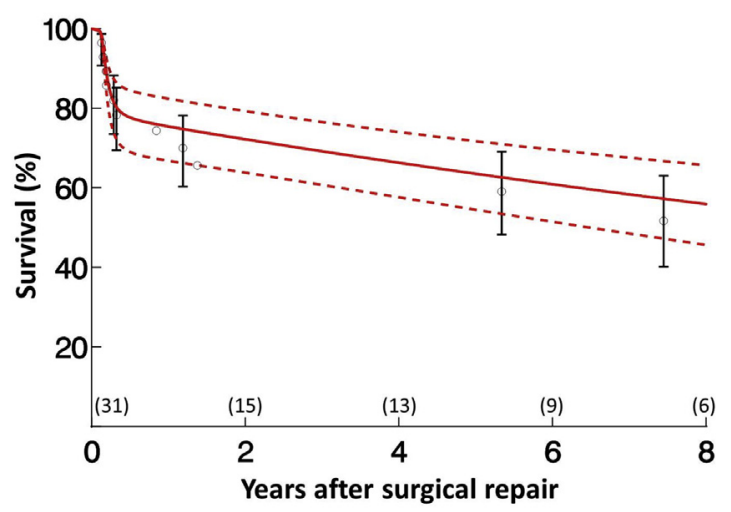

FIGURE 3. Overall estimated survival after repair of PV stenosis. Overall unadjusted 8-year survival for all 31 patients after surgical repair for PV stenosis. The survival was $75 \% \pm 7 \%, 69 \% \pm 8 \%$ and $64 \% \pm 7 \%$ at 1,3 , and 5 years, respectively. By 8 years, survival decreases to $56 \% \pm 9 \%$. Independent of time, there were a total of 11 deaths. Each circle represents a death positioned on the vertical axis by the KaplanMeier estimator. Vertical bars are confidence limits equivalent to \pm 1 standard error. Solid lines are parametric estimates enclosed within dashed $68 \%$ confidence bands equivalent to \pm 1 standard error. Numbers in parentheses are the number of patients at risk at each associated time.

were associated with poor early ( $<1$ year) survival (Table 3 and Figure 4, $A$ and $B$ ). Furthermore, a smaller downstream TCSAi $(P=.059)$ tended to be associated with poor late survival (>1 year) (Figure 4,C), without reaching statistical significance. Of note, none of the different PV morphologies (based on upstream/downstream PVCSAi ratio) were found to influence survival.

TABLE 3. Incremental risk factors for freedom from death

\begin{tabular}{ccccc}
\hline Variables & $\begin{array}{c}\text { Parameter } \\
\text { estimates }\end{array}$ & $\begin{array}{c}\text { Standard } \\
\text { error }\end{array}$ & $\begin{array}{c}\boldsymbol{P} \\
\text { value }\end{array}$ & Reliability* \\
\hline $\begin{array}{c}\text { Early phase }(<1 \mathrm{y}) \\
\quad \text { Upstream TCSAi } \\
\left(\mathrm{mm}^{2} / \mathrm{m}^{2}\right)\end{array}$ & -0.008 & 0.004 & .030 & $53 \%$ \\
$\begin{array}{c}\text { No. of stenotic PVs } \\
\text { Constant phase }(>1 \mathrm{y}) \\
\quad \begin{array}{c}\text { Downstream TCSAi } \\
\left(\mathrm{mm}^{2} / \mathrm{m}^{2}\right) \dagger\end{array}\end{array}$ & -0.013 & 0.007 & .059 & $45 \%$ \\
\hline
\end{tabular}

Incremental risk factors for freedom from death for all 31 patients after PV stenosis repair. TCSA $i$, Total cross-sectional area indexed to body surface area; $P V$, pulmonary vein. *Reliability was determined by bootstrap resampling $(\mathrm{n}=500)$. $\dagger$ Although it only approached significance, the downstream TCSAi variable was manually included in the final multivariable model to illustrate the impact that downstream vessel size has on late survival. On univariate analysis, both upstream TCSAi (parameter estimate $=-0.014 ; P$ early $=.048$ ) and number of stenotic PVs (parameter estimate $=1.5 ; P$ early $=.020$ ) were significant risk factors for death, whereas downstream TCSAi only approached significance on univariate analysis (parameter estimate $=-0.013 ; P$ constant $=.055$ ). Furthermore, if the model was reproduced excluding the downstream TCSAi, both upstream TCSAi and number of stenotic PVs remained significant risk factors for death. Parameter estimates were determined from multiphase risk hazard analysis and represent the magnitude of a risk factor for death. 
Survival (1-year) stratified by Upstream TCSAi vs Downstream TCSAi

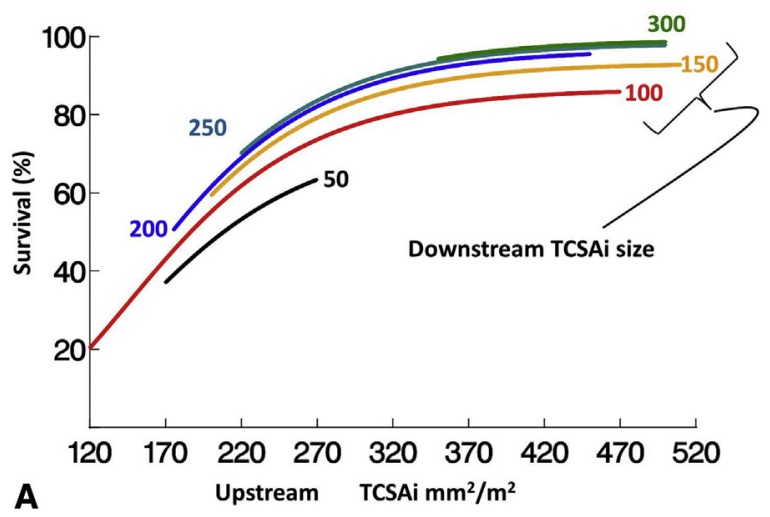

Survival stratified by Upstream TCSAi versus Downstream TCSAi (2 PVs disease, median upstream TCSAi $=260 \mathrm{~mm}^{2} / \mathrm{m}^{2}$ )

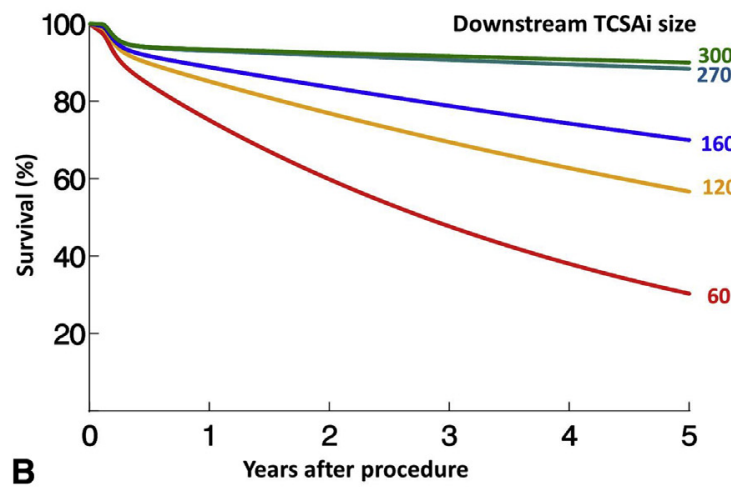

Survival by number of stenotic pulmonary veins (median upstream TCSAi $=260 \mathrm{~mm}^{2} / \mathrm{m}^{2}-$ median downstream TCSAi $=160 \mathrm{~mm}^{2} / \mathrm{m}^{2}$ )

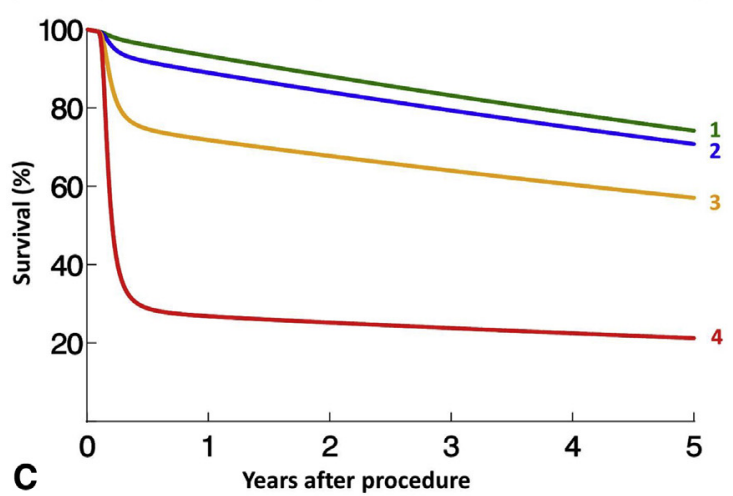

FIGURE 4. The impact of upstream TCSAi, downstream TCSAi, and number of stenotic/occluded PVs on survival. A, Risk-adjusted nomogram demonstrating the differential impact on survival across the spectrum of upstream and downstream PV dimensions. The estimated 1-year survival is on the vertical axis across the spectrum of upstream TCSAi. Each curve represents the 1-year survival for a hypothetical patient with a given downstream TCSAi dimension. The black curve represents a downstream TCSAi of $50 \mathrm{~mm}^{2} / \mathrm{m}^{2}$. The red curve represents a downstream TCSAi of $100 \mathrm{~mm} / \mathrm{m}^{2}$. The yellow curve represents a downstream TCSAi of $150 \mathrm{~mm}^{2} / \mathrm{m}^{2}$. The blue curve represents a downstream TCSAi of $200 \mathrm{~mm}^{2} / \mathrm{m}^{2}$. The teal curve represents a downstream TCSAi of $250 \mathrm{~mm}^{2} / \mathrm{m}^{2}$. The green curve represents a downstream TCSAi of

\section{Restenosis and Reoperation}

Among the 28 hospital survivors, restenosis occurred in 10 patients $(37.5 \%), 7$ of whom did not undergo further surgery. Among these 7 patients, 3 are alive at last follow-up and 4 died secondary to disease progression. Three patients with PV restenosis underwent subsequent procedures, including revision of the right-sided sutureless repair $(\mathrm{n}=1)$, pneumectomy $(\mathrm{n}=1)$, and stent implantation $(\mathrm{n}=1)$. The first 2 patients are alive as of the last follow-up. The patient who underwent stent implantation was diagnosed with congenital PV stenosis and complete occlusion of the LUPV and subsequently underwent complete sutureless repair. This resulted in minimal clinical improvement and elevated right ventricular pressure that became equivalent to systemic pressure 2 months postrepair. Postrepair CT confirmed the persistence of RUPV- RLPV and LLPV severe hypoplasia and occlusion of the LUPV. LLPV stenting was performed as a salvage procedure to reduce the right ventricular pressures. Although LLPV stenting provided temporary improvement and resulted in hospital discharge, ultimately, pulmonary hypertension and severe right ventricular dysfunction resulted in death 2 months postdischarge.

\section{DISCUSSION}

PV stenosis scoring systems have typically categorized the severity of stenosis using subjective echocardiographic data. ${ }^{4,10,11}$ In this series, we used CT/MRI measurements to obtain normalized PV dimensions as indicators of stenosis.

$300 \mathrm{~mm}^{2} / \mathrm{m}^{2}$. The values of the upstream TCSAi have been truncated to fit within the range of data for a given downstream TCSAi size. For example, a patient with a downstream TCSAi of $100 \mathrm{~mm} / \mathrm{m}^{2}$ (red curve) could have an upstream TCSAi from 120 to $470 \mathrm{~mm}^{2} / \mathrm{m}^{2}$, whereas a patient with a downstream TCSAi of $50 \mathrm{~mm}^{2} / \mathrm{m}^{2}$ (black curve) only had an upstream TCSAi between 170 and $270 \mathrm{~mm}^{2} / \mathrm{m}^{2}$. B, The estimated 5-year adjusted survival demonstrating the impact of the downstream TCSAi size on survival. The late survival ( $>1$ year) tended to be worse for smaller downstream TCSAi. Each curve represents a hypothetical patient with 2-vessel stenosis and a median upstream TCSAi size of $260 \mathrm{~mm}^{2} / \mathrm{m}^{2}$. Each curve represents the estimated survival based on parametric estimates stratified by the size of the downstream TCSAI: red curve $=60 \mathrm{~mm}^{2} / \mathrm{m}^{2}$; yellow curve $=120 \mathrm{~mm}^{2} / \mathrm{m}^{2} ;$ blue curve $=160 \mathrm{~mm}^{2} / \mathrm{m}^{2}$; teal curve $=270 \mathrm{~mm}^{2} / \mathrm{m}^{2} ;$ green curve $=300 \mathrm{~mm}^{2} / \mathrm{m}^{2}$. C, The estimated 5 -year adjusted survival demonstrating the impact of number total number of stenotic PVs on survival. A greater number of PVs with stenosis results in significantly worse survival. The differences in survival are related predominantly to a poor early survival ( $<1$ year) that results with increased vessel involvement. Each curve represents the estimated survival for a hypothetical patient (based on parametric estimates) stratified by the number of stenotic veins with a median upstream TCSAi size of $260 \mathrm{~mm}^{2} / \mathrm{m}^{2}$ and median downstream TCSAi size of $160 \mathrm{~mm}^{2} / \mathrm{m}^{2}$ : red curve $=4$ veins with stenosis; yellow curve $=3$ veins with stenosis; blue curve $=2$ veins with stenosis; green curve $=1$ vein with stenosis. TCSA $i$, Total pulmonary vein cross-sectional area indexed to body surface area. 
Of note, there are minimal published data ${ }^{14,21}$ that define the dimensions of a PV that constitutes a stenotic vein. In the present study, we demonstrated that a downstream PVCSAi less than $50 \mathrm{~mm}^{2} / \mathrm{m}^{2}$ relates to the conventional clinical assessments of stenosis (with both preoperative imaging and surgical reports) and diminished PV blood flow. Upstream PV dimensions are obscured by the lung parenchyma when evaluated via echocardiography, but they are obtainable by CT/MRI. Therefore, CT/MRI imaging allows a more comprehensive and accurate determination of the extent of PV stenosis compared with traditional echocardiographic imaging. ${ }^{12-16}$ As such, a predictive system that incorporates these dimensions is likely to have better prognostic capabilities. It has to be considered that a systematic difference in dimension assessment of PVs between CT and MRI can be present in our series, but because of the lack of published evidence to support the existence of this difference, especially for the pediatric population, we have accepted the interchangeability of the two diagnostic modalities.

In prior studies, severity of PV stenosis has been assessed using echocardiogram pressure gradients measured in each vein. ${ }^{4,11}$ However, measurement of gradients can lead to underestimation of the stenosis severity because of lowvelocity blood flow related to flow redistribution toward the less-obstructed portions of the lung. ${ }^{14,22}$ In unilateral PV stenosis, flow redistribution is characterized by holodiastolic flow reversal in the ipsilateral pulmonary artery ${ }^{14}$ and greater flow to nonstenotic PVs. ${ }^{22}$ In the present study, pulmonary artery flow redistribution occurred in $65 \%$ of the patients. Flow redistribution may represent a compensatory response to redirect blood flow from stenotic PVs to the lower-resistance lung segments. We postulate that blood redistribution may increase shear forces by increasing the flow in nonstenotic PVs and may promote local endothelial cell activation as a response to increase local flow. $^{23,24}$ Activated endothelial cells can acquire a mesenchymal transitional phenotype ${ }^{25,26}$ with an increase in extracellular matrix deposition in the lesion area. ${ }^{27}$ Thus, we speculate that augmented shear stress (as a result of flow redistribution) could initiate endothelial mesenchymal transition and disease propagation in unobstructed PVs.

Segmental dimensions (upstream and downstream) and type of stenosis (upstream/downstream ratio) are important when considering management strategies for PV stenosis. A limitation of the surgical repair is the inability to address stenosis that extends deeply into the intraparenchymal upstream segments. ${ }^{1,28,29}$ We hypothesize that this explains why some postrepair stenosis and the majority of patients with congenital stenosis have poor survival after surgery. ${ }^{1,3-6}$ Support for the importance of smaller upstream dimensions is underscored by several studies in which patients with diffusely small PVs do worse than those with discrete stenosis. ${ }^{5,6}$

Because surgical repair is unable to access deeply into the upstream PVs, we need to develop pharmacologic agents targeting PV pathology. ${ }^{26,30}$ Testing of pharmacologic treatments for upstream PV stenosis will be challenging because of the relatively low incidence of this disease in any single center. Because a multicenter trial will likely be required to test a pharmacologic therapy, it will be crucial to develop risk-stratification algorithms to identify high-risk subsets of patients with PV stenosis, because this subset will be most likely to demonstrate benefit (or lack of benefit) when treated in a clinical trial.

Risk stratification for patients with PV stenosis is currently challenging because of the variability in the anatomic configuration and the unknown relationship between these anatomic variations and survival. Our study demonstrates that by using cross-sectional areas (PVCSAi and TCSAi) and tabulating the number of stenotic PVs, we can identify high-risk subsets of patients with high predicted mortality. As secondary end points, it also may be possible (although beyond the scope of the current study) to monitor upstream PV dimensions and flow redistribution using MRI to characterize the response to systemic therapies directed at PV stenosis.

\section{Severity of Disease: Number of Stenotic Pulmonary Veins and Upstream and Downstream Cross- Sectional Areas}

A greater number of stenotic PVs has been shown to be associated with an increased risk of death, especially when there is bilateral involvement. ${ }^{5-9}$ Patients with bilateral stenosis are subjected to more severe PV hypertension compared with patients with unilateral disease likely because they lost the capacity to redistribute flow as a compensatory mechanism. Greater PV hypertension has been found to be associated with more severe thickening of the pulmonary artery media $^{31,32}$ and more capillary remodeling ${ }^{33,34}$ that could lead to severe pulmonary arterial hypertension. Each PV involved will determine an increment of pulmonary resistance directly related to the extent of the narrowing and a loss of compensatory flow redistribution related to the number of veins affected.

Smaller upstream TCSAi was associated with poor survival in the first year after repair. We hypothesize that this is related to the inability to surgically relieve the upstream obstruction. Furthermore, smaller upstream TCSAi may be a marker of more aggressive forms of stenosis with more extensive pulmonary venous vasculopathy. ${ }^{25,26,35}$ Therefore, upstream TCSAi assessed by CT or MRI may be helpful in guiding preoperative counseling and decision making. 
Smaller downstream TCSAi is associated with poor long-term survival. Although stenosis at the left atrial junction can be effectively relieved with surgery, ${ }^{4,28,29}$ it is possible that more severe stenosis at the time of repair sets in motion a biologic process that increases the risk of postrepair PV stenosis as noted in human studies ${ }^{1,5,6,8}$ and animal models. ${ }^{25,26}$ Recurrent PV stenosis can occur in isolated or multiple PVs. In either case, recurrent stenosis can progress with minimal hemodynamic derangement in the early stages of disease. Consequently, our data suggest that patients with smaller downstream TCSAi should be monitored aggressively in the years after repair.

\section{Study Limitations}

Our study is limited by being a retrospective analysis of a small cohort of patients. In addition, we are limited in our ability to look at the postoperative progression of disease because of the limited number of CT/MRI images at standard time points. We also acknowledge that the study does not include extremely low birth weight and premature patients because of the difficulties to perform CT/MRI on them.

\section{CONCLUSIONS}

We defined an objective methodology for classifying PV stenosis that captures both downstream and upstream dimensions and describes mode of stenosis. The upstream total cross-sectional area and the number of stenotic PVs influence early survival and can be used to guide counseling. Smaller downstream cross-sectional area influences late survival, and those patients should be monitored with close follow-up. This methodology could aid in risk stratification for future clinical trials of pharmacologic agents designed to target upstream pulmonary vasculopathy.

\section{Conflict of Interest Statement}

Authors have nothing to disclose with regard to commercial support.

You can watch a Webcast of this AATS meeting presentation by going to: http://webcast.aats.org/2015/Video/ Tuesday/04-28-15_6A_1615_Lo_Rito.mp4.

\section{References}

1. Caldarone CA, Najm HK, Kadletz M, Smallhorn JF, Freedom RM, Williams WG, et al. Relentless pulmonary vein stenosis after repair of total anomalous pulmonary venous drainage. Ann Thorac Surg. 1998;66:1514-20.

2. Seale AN, Uemura H, Webber SA, Partridge J, Roughton M, Ho SY, et al. Total anomalous pulmonary venous connection: morphology and outcome from an international population-based study. Circulation. 2010;122:2718-26.

3. Hancock Friesen CL, Zurakowski D, Thiagarajan RR, Forbess JM, del Nido PJ, Mayer JE, et al. Total anomalous pulmonary venous connection: an analysis of current management strategies in a single institution. Ann Thorac Surg. 2005; 79:596-606.

4. Viola N, Alghamdi AA, Perrin DG, Wilson GJ, Coles JG, Caldarone CA. Primary pulmonary vein stenosis: the impact of sutureless repair on survival. $J$ Thorac Cardiovasc Surg. 2011;142:344-50.
5. Seale AN, Webber SA, Uemura H, Partridge J, Roughton M, Ho SY, et al. Pulmonary vein stenosis: the UK, Ireland and Sweden collaborative study. Heart. 2009;95:1944-9.

6. Seale AN, Uemura H, Webber SA, Partridge J, Roughton M, Ho SY, et al. Total anomalous pulmonary venous connection: outcome of postoperative pulmonary venous obstruction. J Thorac Cardiovasc Surg. 2013;145:1255-62.

7. Song MK, Bae EJ, Jeong SI, Kang IS, Kim NK, Choi JY, et al. Clinical characteristics and prognostic factors of primary pulmonary vein stenosis or atresia in children. Ann Thorac Surg. 2013;95:229-34.

8. Holt DB, Moller JH, Larson S, Johnson MC. Primary pulmonary vein stenosis. Am J Cardiol. 2007;99:568-72.

9. Breinholt JP, Hawkins JA, Minich L, Tani LY, Orsmond GS, Ritter S, et al. Pulmonary vein stenosis with normal connection: associated cardiac abnormalities and variable outcome. Ann Thorac Surg. 1999;68:164-8.

10. Jenkins KJ, Sanders SP, Orav EJ, Coleman EA, Mayer JE, Colan SD. Individual pulmonary vein size and survival in infants with totally anomalous pulmonary venous connection. J Am Coll Cardiol. 1993;22:201-6.

11. Yun T-J, Coles JG, Konstantinov IE, Al-Radi OO, Wald RM, Guerra V, et al. Conventional and sutureless techniques for management of the pulmonary veins: evolution of indications from postrepair pulmonary vein stenosis to primary pulmonary vein anomalies. J Thorac Cardiovasc Surg. 2005;129: 167-74.

12. Grosse-Wortmann L, Al-Otay A, Goo HW, Macgowan CK, Coles JG, Benson LN, et al. Anatomical and functional evaluation of pulmonary veins in children by magnetic resonance imaging. J Am Coll Cardiol. 2007;49: 993-1002.

13. Greenway SC, Yoo S-J, Baliulis G, Caldarone C, Coles J, Grosse-Wortmann L. Assessment of pulmonary veins after atrio-pericardial anastomosis by cardiovascular magnetic resonance. J Cardiovasc Magn Reson. 2011;13:72.

14. Roman KS, Kellenberger CJ, Macgowan CK, Coles J, Redington AN, Benson LN, et al. How is pulmonary arterial blood flow affected by pulmonary venous obstruction in children? A phase-contrast magnetic resonance study. Pediatr Radiol. 2005;35:580-6.

15. Valsangiacomo ER, Levasseur S, McCrindle BW, MacDonald C, Smallhorn JF, Yoo S-J. Contrast-enhanced MR angiography of pulmonary venous abnormalities in children. Pediatr Radiol. 2003;33:92-8.

16. Valsangiacomo ER, Barrea C, Macgowan CK, Smallhorn JF, Coles JG, Yoo SJ. Phase-contrast MR assessment of pulmonary venous blood flow in children with surgically repaired pulmonary veins. Pediatr Radiol. 2003;33: $607-13$.

17. Haycock GB, Schwartz GJ, Wisotsky DH. Geometric method for measuring body surface area: a height-weight formula validated in infants, children, and adults. J Pediatr. 1978;93:62-6.

18. Sluysmans T, Colan SD. Theoretical and empirical derivation of cardiovascular allometric relationships in children. J Appl Physiol. 2005;99:445-57.

19. Blackstone EH, Naftel DC, Turner ME. The decomposition of time-varying hazard into phases, each incorporating a separate stream of concomitant information. J Am Stat Assoc. 1986;81:615-24.

20. Breiman L. Bagging predictors. Mach Learn. 1996;24:123-40.

21. Dill T, Neumann T, Ekinci O, Breidenbach C, John A, Erdogan A, et al. Pulmonary vein diameter reduction after radiofrequency catheter ablation for paroxysmal atrial fibrillation evaluated by contrast-enhanced three-dimensional magnetic resonance imaging. Circulation. 2003;107:845-50.

22. Kotani Y, Zhu J, Grosse-Wortmann L, Honjo O, Coles JG, Van Arsdell GS, et al. Anatomical risk factors, surgical treatment, and clinical outcomes of left-sided pulmonary vein obstruction in single-ventricle patients. J Thorac Cardiovasc Surg. 2015;149:1332-8.

23. Park JES, Lyon AR, Shao D, Hector LR, Xu H, O'Gara P, et al. Pulmonary venous hypertension and mechanical strain stimulate monocyte chemoattractant protein-1 release and structural remodelling of the lung in human and rodent chronic heart failure models. Thorax. 2014;69:1120-7.

24. Conway DE, Williams MR, Eskin SG, McIntire LV. Endothelial cell responses to atheroprone flow are driven by two separate flow components: low time-average shear stress and fluid flow reversal. Am J Physiol Heart Circ Physiol. 2010;298: H367-74.

25. Kato H, Fu YY, Zhu J, Wang L, Aafaqi S, Rahkonen O, et al. Pulmonary vein stenosis and the pathophysiology of "upstream" pulmonary veins. J Thorac Cardiovasc Surg. 2014;148:245-53.

26. Zhu J, Ide H, Fu YY, Teichert A-M, Kato H, Weisel RD, et al. Losartan ameliorates "upstream" pulmonary vein vasculopathy in a piglet model of pulmonary vein stenosis. J Thorac Cardiovasc Surg. 2014;148:2550-7. 
27. LaBourene JI, Coles JG, Johnson DJ, Mehra A, Keeley FW, Rabinovitch M. Alterations in elastin and collagen related to the mechanism of progressive pulmonary venous obstruction in a piglet model. A hemodynamic, ultrastructural, and biochemical study. Circ Res. 1990;66:438-56.

28. Hickey EJ, Caldarone CA. Surgical management of post-repair pulmonary vein stenosis. Semin Thorac Cardiovasc Surg Pediatr Card Surg Annu. 2011;14:101-8.

29. Lacour-Gayet F. Surgery for pulmonary venous obstruction after repair of total anomalous pulmonary venous return. Pediatr Card Surg Annu. 2006;9:45-50.

30. Rehman M, Jenkins KJ, Juraszek AL, Connor JA, Gauvreau K, Muneeb M, et al. A prospective phase II trial of vinblastine and methotrexate in multivessel intraluminal pulmonary vein stenosis in infants and children. Congenit Heart Dis. 2011;6:608-23.

31. Yamaki S, Tsunemoto M, Shimada M, Ishizawa R, Endo M, Nakayama S, et al. Quantitative analysis of pulmonary vascular disease in total anomalous pulmonary venous connection in sixty infants. J Thorac Cardiovasc Surg. 1992;104:728-35.

32. Endo M, Yamaki S, Ohmi M, Tabayashi K. Pulmonary vascular changes induced by congenital obstruction of pulmonary venous return. Ann Thorac Surg. 2000; 69:193-7.

33. Guazzi M, Arena R. Pulmonary hypertension with left-sided heart disease. Nat Rev Cardiol. 2010;7:648-59.

34. Kay JM, Edwards FR. Ultrastructure of the alveolar-capillary wall in mitral stenosis. J Pathol. 1973;111:239-45.

35. Lathen C, Zhang Y, Chow J, Singh M, Lin G, Nigam V, et al. ERG-APLNR axis controls pulmonary venule endothelial proliferation in pulmonary veno-occlusive disease. Circulation. 2014;130:1179-91.

Key Words: congenital heart disease, pulmonary vein stenosis, magnetic resonance imaging, computed tomography, predictive model, survival

\section{Discussion}

Dr F. Lacour-Gayet (London, $U K$ ). Your study proposed a detailed assessment of the morphology of the PV stenosis based on CT scan and MRI. The diameter of the stenosis was indexed on the body surface area and calculated at 3 points: at the level of the left atrial junction downstream, at the level of the last venous branching in the lung parenchyma upstream, and at the maximum point of stenosis. The comprehensive quantification of full PV stenosis is followed by a sophisticated risk analysis. Two thirds of the 31 patients have a native PV stenosis isolated or associated with congenital heart disease, and one third have secondary PV stenosis after total vein or other cardiac surgery. All patients but 1 underwent a sutureless repair. The hospital mortality is $10 \%$, and survival at 5 years is $64 \%$. This is the third article this afternoon on PV stenosis. It appears that sutureless repair is the surgical technique most frequently used; however, the results are grim, and PV stenosis continues to stand probably as the most mysterious and frustrating congenital heart disease today. I have 4 questions.

In the article and in your presentation, you don't mention selective pulmonary artery angiography. Do you consider CT scan and MRI as the optimal imaging methods to evaluate PV stenosis today?

Dr Lo Rito. Yes, I believe that CT and MRI should be used routinely to assess PV stenosis because they are the optimal imaging available. The majority of patients with PV stenosis usually undergo to surgery with an echocardiogram only. But if we can perform a CT or MRI, we can assess the part of the vein that is not possible to visualize by echocardiogram, like the upstream segment, and that seems to be the strongest predictor for early survival. CT and MRI can then help to stratify patients and counsel the family and give the appropriate prediction of survival after the surgery we will perform.

Dr Lacour-Gayet. But do you still do a catheterization or not?

Dr Lo Rito. No, we do not perform catheterization in all the patients.

Dr Lacour-Gayet. The median weight of patients in your series is $6 \mathrm{~kg}$, and the smallest patient weighs $4.5 \mathrm{~kg}$. So it looks like premature small babies are excluded from your study. Is the reason that there is some limitation an indication of CT scan or MRI in a small weight population?

Dr Lo Rito. Yes, I think the premature low weight baby represents a limitation for CT and MRI. CT and MRI techniques changed dramatically, especially during the time frame of this study (1992-2012). New methodology to perform CT and MRI in smaller patients have developed in the last years moving to a lower body weight. The study is limited only to those who underwent to CT and MRI, so they are the patients in whom it was possible to perform CT/MRI and then they were at larger weight. But we don't want to translate this to the whole population with PV stenosis because we have a selected group of patients. A larger study is required to validate our findings.

Dr Lacour-Gayet. But you admit that in doing so you exclude what we are seeing as the worst population presented earlier in the afternoon?

Dr Lo Rito. Yes, I agree that we exclude the premature low weight patients and maybe the worst patients, but the purpose of the study is to find a reliable objective methodology to assess PV stenosis. I believe that if we can apply our method to this group, we will have similar findings.

Dr Lacour-Gayet. Do you find any difference in prognosis between native PV and acquired PV stenosis after total vein surgery in your series? Do you have any data on that?

Dr Lo Rito. I tested the variable congenital and acquired, because congenital PV stenosis is known to be more lethal than acquired PV stenosis. The results were not significant in my analysis. By selecting only the patients who had CT and MRI, then we cannot definitely exclude differences between acquired and congenital forms.

Dr Lacour-Gayet. Finally, as you have noticed, we're all surprised that what I will call the good forms, the ones with downstream stenosis, meaning their stenosis is at the level of the left atrium, don't have very good results, correct, that's what you have observed?

Dr Lo Rito. In the long term, yes.

Dr Lacour-Gayet. Is it possible that the surgical technique can be the cause of this observation? I recommend to resect all this area, and others have 
recommended just to patch this area. Do you think it could be possible that leaving some pathologic tissue in place could explain the long-term stenosis?

Dr Lo Rito. Currently, our strategy is to resect as much as possible of the stenotic tissue. So as surgeons, we think, okay, we have solved the problem because we resected, but clearly we didn't solve it. Our hypothesis to explain this surprising finding about downstream is the following. Patients with downstream stenosis might develop a progressive disease that will carry on across the year. Because we cannot detect the exact changes on echocardiogram, maybe when we detect a significant gradient on echocardiogram it's too late for these patients because the disease has progressed inside the lung. That's the hypothesis we have.

Dr C. Baird (Boston, Mass). Excellent presentation, and obviously data like these will help us progress. I agree with the comment about CT or MRI. We've turned to that to try to assess these veins as well.

Is there any way you can account for collaterals? Because one of the things we have seen is collaterals developed with veins that ultimately become atretic. If there are large collaterals, we suspect those patients have actually done better, although I don't have any data right now to support that. So is there any way to account for large collaterals that develop?

Dr Lo Rito. Well, for those 31 patients, the images were reviewed by 2 expert cardiac radiologists. They didn't report the presence of collaterals. But I cannot exclude their existence because those 31 patients were from a cohort of 145 patients, so we have more than two thirds who did not receive CT or MRI who could have collaterals.

Dr Baird. I think it's excellent, and obviously it's the first round of trying to define how we look at this. Maybe as a second part, we can figure out how to include the collateralization in there.

Dr Lo Rito. That's a great suggestion, and probably we should count for collaterals.
Dr Baird. Second, your grading system, how does it account for patients whether they had surgery or not and the aggressiveness of the surgery? So your grading system predicts death, right?

Dr Lo Rito. Our system doesn't account for the extent of surgery because we already resect as much as possible in these children, and the resection cannot be extended further. The purpose of our system is to create a tool that can allow you, before those patients undergo surgery, to understand how they will perform after. We believe that the upstream portion of the PV is the most important because it's what we cannot treat surgically. We cannot assess that with echocardiogram. This is probably the reason why those patients perform badly after the operation.

Dr Baird. So did all of these patients undergo operation?

Dr Lo Rito. Yes, all 31 patients underwent surgery and had preoperative CT and MRI.

Dr Baird. To simplify, do you think taking a single measurement at the point of maximal stenosis may yield the same predictions?

Dr Lo Rito. We test the point of maximal stenosis. It coincides in two thirds of the patients to the downstream portion, so we cannot highlight that. I believe that the total cross-sectional area is the most important measurement, and it's based on a physics rule about fluid dynamics: The smaller the cross-section, the higher the gradient. A higher gradient is going to be reflected in a greater pressure in the right ventricle and the higher the chance to fail later. So we believe that the total cross-sectional area is what is driving the survival.

Dr C. Huddleston (St Louis, Mo). I was struck by the survival curve where you had some patients with 1 or 2 veins involved but clearly don't have a normal survival. A patient who has only a single stenotic PV should have almost normal survival, unless something else is going on. Again, that sort of reiterates my fatalistic view of this disease, but I enjoyed the presentation.

Dr Lo Rito. Thank you. 


\section{APPENDIX E1. STATISTICAL METHODS Parametric Risk Hazard Analysis}

Parametric risk hazard analysis was used to identify incremental risk factors from the date of initial pulmonary vein (PV) repair to the date of death, with patients otherwise censored at the last known date to be alive. Specifically, our aim was to address how the magnitude and location of PV stenosis influences survival after PV repair. In preliminary analyses, we evaluated end states other than death, including invasive reintervention and restenosis. However, given the relatively few reinterventions to date, our final results focused strictly on survival. In addition, because the evolution of vessels stenosis is a dynamic process, determining the "exact" time that restenosis occurs is challenging. Therefore, using freedom from restenosis as a time-related end state is not practical or valid, and thus we limited our restenosis results to a descriptive evaluation.

Parametric hazard models use the underling hazard function based on unadjusted Kaplan-Meier estimates of the time to event. Parametric models are tested for adequate fit of the underlying data by plotting observed Kaplan-Meier estimates versus the predicted (expected) parametric events over time. Subsequently, each parametric model, which uses shaping parameters that capture various "phases of risk," can be independently subjected to risk hazard regression analysis. ${ }^{19}$ (For additional details, see http://my.clevelandclinic.org/ professionals/software/hazard/default.aspx.)

\section{Risk Hazard Analysis}

Each parametric hazard function can be subject to risk hazard analysis. To minimize the risk of model over determination, continuous variables with excessive missing values $(>75 \%)$ were excluded. Variable selection was guided by bootstrap bagging ( $\mathrm{n}=500$, threshold for inclusion $P<.1$; threshold for retention $P<.06) .{ }^{16}$ Variables selected in at least approximately $50 \%$ of re-samples or their clusters were considered reliable for inclusion in a multivariable stepwise regression analysis. To create the final risk-adjusted model, parametric hazard analysis was performed on bootstrap-proven variables. Important variables of interest (ie, PV dimensions) were sometimes included in the analysis with a $P$ value greater than .05 to illustrate their relative impact on survival. 
TABLE E1. Univariate analysis of preoperative characteristics

\begin{tabular}{lccc}
\hline \multicolumn{1}{c}{ Continuous Variables } & Mean + SD & $\begin{array}{c}\text { Parameter } \\
\text { estimate }\end{array}$ & $\boldsymbol{P}$ value \\
\hline Age at operation $(\mathrm{d})$ & $793 \pm 1460$ & -0.016 & .033 \\
BSA $\left(\mathrm{m}^{2}\right)$ & $0.45 \pm 0.32$ & -19 & .030 \\
TCSAi downstream $\left(\mathrm{mm}^{2} / \mathrm{m}^{2}\right)$ & $194 \pm 109$ & -0.013 & .053 \\
TCSAi upstream $\left(\mathrm{mm}^{2} / \mathrm{m}^{2}\right)$ & $302 \pm 122$ & -0.014 & .048 \\
Aortic area indexed $\left(\mathrm{mm}^{2} / \mathrm{m}^{2}\right)$ & $421 \pm 152$ & -0.002 & .61 \\
\hline & & & \\
\multicolumn{1}{c}{ Categoric variables } & No. of patients & Parameter & \\
\hline Gender (male/female) & $23 / 8$ & 0.61 & .51 \\
Associated syndrome (yes/no) & $5 / 26$ & -0.033 & .97 \\
No. of stenotic PVs (1-4) & & 1.5 & .020 \\
Bilateral PV stenosis (yes/no) & $14 / 17$ & 2.9 & .065 \\
\hline
\end{tabular}

Descriptive statistics for univariate analysis: mean with standard deviation or number of patients with the parameter estimate and $P$ value for each variable. $S D$, Standard deviation; $B S A$, body surface area; $T C S A i$, total cross-sectional area indexed to body surface area; $P V$, pulmonary vein. 